\title{
TU/e EmonONEN

\section{Sufficient conditions for data-driven stability of ellipsoidal unfalsified control}

\section{Citation for published version (APA):}

Helvoort, van, J. J. M., Jager, de, A. G., \& Steinbuch, M. (2006). Sufficient conditions for data-driven stability of ellipsoidal unfalsified control. In Proceedings of the 45th IEEE Conference on Decision \& Control, San Diego, CA, USA, December 13-15, 2006 (pp. 453-458). Institute of Electrical and Electronics Engineers. https://doi.org/10.1109/CDC.2006.376826

DOI:

10.1109/CDC.2006.376826

Document status and date:

Published: 01/01/2006

\section{Document Version:}

Publisher's PDF, also known as Version of Record (includes final page, issue and volume numbers)

\section{Please check the document version of this publication:}

- A submitted manuscript is the version of the article upon submission and before peer-review. There can be important differences between the submitted version and the official published version of record. People interested in the research are advised to contact the author for the final version of the publication, or visit the $\mathrm{DOI}$ to the publisher's website.

- The final author version and the galley proof are versions of the publication after peer review.

- The final published version features the final layout of the paper including the volume, issue and page numbers.

Link to publication

\section{General rights}

Copyright and moral rights for the publications made accessible in the public portal are retained by the authors and/or other copyright owners and it is a condition of accessing publications that users recognise and abide by the legal requirements associated with these rights.

- Users may download and print one copy of any publication from the public portal for the purpose of private study or research.

- You may not further distribute the material or use it for any profit-making activity or commercial gain

- You may freely distribute the URL identifying the publication in the public portal.

If the publication is distributed under the terms of Article 25fa of the Dutch Copyright Act, indicated by the "Taverne" license above, please follow below link for the End User Agreement:

www.tue.nl/taverne

Take down policy

If you believe that this document breaches copyright please contact us at:

openaccess@tue.nl

providing details and we will investigate your claim. 


\title{
Sufficient Conditions for Data-driven Stability of Ellipsoidal Unfalsified Control
}

\author{
Jeroen van Helvoort, Bram de Jager and Maarten Steinbuch
}

\begin{abstract}
Unfalsified Control is a direct data-driven, plantmodel-free controller design method, which recursively falsifies controllers that fail to meet the required performance specification, making them ineligible to actually control the plant. In this paper it is shown that sufficient conditions for stability can be derived for Unfalsified Control with an ellipsoidal Unfalsified set, Ellipsoidal Unfalsified Control (EUC). These conditions are: feasibility of the adaptive control problem, discarding of demonstrable destabilizing controllers and a finite number of controller switches. The latter is guaranteed by imposing a maximum volume ratio between two consecutive ellipsoidal Unfalsified sets and a minimum stepsize on the controller adjustments.
\end{abstract}

\section{INTRODUCTION}

There are numerous motives to support data-driven control design, e.g., a priori definition of controller complexity, adaptation to specific disturbances, elimination of model mismatch/assumptions. A data-driven control design method focuses on finding a controller, using merely input-output data of the system. Since these methods do not require a plant model, the assumptions and conservatism introduced in the plant modeling step are omitted.

An emerging data-driven control design method is Unfalsified Control [9]. This data-driven, plant-model-free control approach recursively falsifies control parameter sets that fail to satisfy a performance specification, given measured data and a specified control law. Unfalsified Control theory was later employed in, e.g., [2], [3], [5], [8], [11].

Although in early works the parameter space was gridded (resulting in a finite, but often large, set of candidate controllers), this restriction was lifted by applying a quadratic performance specification to a control law, where the control parameters appear affine [2], [3]. As a result, a continuous region of unfalsified control parameter sets can be regarded, hence, with infinitely many controllers. In Ellipsoidal Unfalsified Control [3], the continuous region of controllers is described by an ellipsoid, resulting in simple algebraic equations to describe the entire set (An introduction to Ellipsoidal Unfalsified Control will be provided in Section III).

A crucial element for any control design method is the notion of stability. Especially for a plant-model-free

All authors are with the department of Mechanical Engineering, Control Systems Technology group of the Technische Universiteit Eindhoven, Eindhoven, The Netherlands. j.j.m.v.helvoortatue.nl control design method, no a priori statements can be made whether a specific controller is stabilizing the closed loop system. In [10] it was shown that for adaptive control systems, sufficient conditions for stability can be derived. The conditions imply that the adaptive control problem is feasible, the cost functional is cost-detectable (i.e., the cost goes to infinity if the controller is unstable) and that the number of controller switches is finite. The latter condition is satisfied by imposing a monotone non-decreasing cost functional which is bounded from above and some minimum improvement in the cost functional between two consecutive controller switches. Furthermore, if a continuous set of controllers is regarded, some neighborhood around a controller with similar performance is required (hence, which is falsified simultaneously).

In this paper, the stability results of [4] are extended, to cover a less restrictive controller selection and a proposed general candidate controller class. Sufficient conditions to guarantee the stability of the adaptive control system are given. The conditions can be summarized as: 1) feasibility of the adaptive control problem, 2) discarding of demonstrably destabilizing controllers, and 3) a maximum number of controller switches.

In this paper, it is shown that conditions 2) and 3) are fulfilled for Ellipsoidal Unfalsified Control. Hence, with the basic assumption of feasibility, the Ellipsoidal Unfalsified Control adaptive system is guaranteed to be stable.

\section{PRELIMINARIES}

As in [1], an adaptive controller is defined as a controller with adjustable parameters and a mechanism for adjusting the parameters. An adaptive control system is a control system with an adaptive controller. The candidate controller set is the set composed by time-invariant controllers with any of the possible parameters [12]. The adaptive control problem is feasible, if the goals for the adaptive control system (e.g., stabilization, performance) are satisfied by a region $E$ of controllers in the (continuously distributed) candidate controller set. Region $E$ has a volume of at least $e>0$, but is unknown a priori.

As in [6], a controller is considered Stably Causally-LeftInvertible (SCLI), if the mapping from the measurement data to the corresponding fictitious reference signal (see Section III-C) is causal and stable [6, Definition 9], i.e., if the fictitious reference signal is uniquely determined by past and present measurement data and if this mapping is stable. 


\section{ELLIPSOIDAL UNFALSIFIED CONTROL}

In this research, the Ellipsoidal Unfalsified Control (EUC) approach is considered, as developed in [3]. In this section, an overview of this data-driven, plant-model-free controller design method is given.

\section{A. Data Acquisition}

The only "plant information" required by EUC is measurement data. The EUC algorithm is applied each time new measurement data becomes available.

\section{B. Candidate Controllers}

A "cloud" of candidate controllers is selected, the candidate controller set. When measurement data is available, candidate controllers might get falsified and the approximation of the set of currently unfalsified controllers, the Unfalsified set, is used as candidate controller set.

Definition 1: The True Unfalsified set is the set of controllers, which are currently unfalsified by all available measurement data.

Definition 2: The Unfalsified set is the approximation of the True Unfalsified set.

The need for gridding of the candidate controllers is overcome by describing the Unfalsified set with a continuous region. In Ellipsoidal Unfalsified Control, the Unfalsified set is defined by an ellipsoid, see [2], which allows for the evaluation of the entire set with simple algebraic equations. The Unfalsified set at time $t_{k-1}$ is described by the ellipsoid

$\mathcal{E}\left(t_{k-1}\right)=$

$$
\left\{\theta \mid\left(\theta-\theta_{c}\left(t_{k-1}\right)\right)^{T} \Sigma^{-1}\left(t_{k-1}\right)\left(\theta-\theta_{c}\left(t_{k-1}\right)\right) \leq 1\right\},
$$

with $\theta \in \mathbb{R}^{p}$ the controller parameters, $\theta_{c}\left(t_{k-1}\right) \in \mathbb{R}^{p}$ the center of the ellipsoid and $\Sigma\left(t_{k-1}\right) \in \mathbb{R}^{p \times p}$ the matrix, which describes the shape of the ellipsoid.

\section{Fictitious Reference}

The fictitious reference is a fictitious signal, used to evaluate the performance of a controller. For a given controller, the fictitious reference is constructed by analyzing which reference would have resulted in exactly the measured input and output of the plant, if that controller would have been implemented.

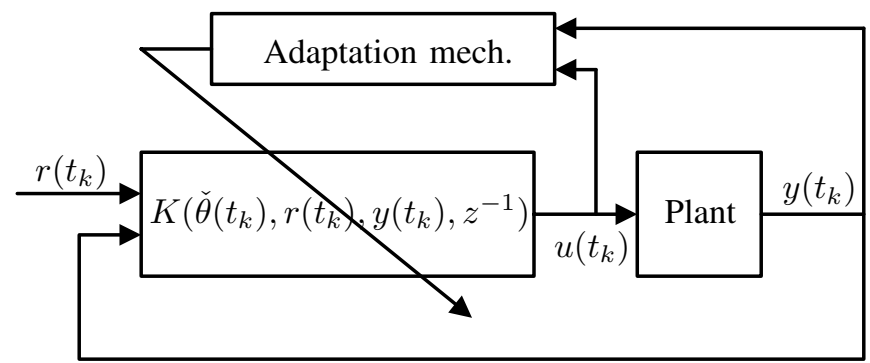

Fig. 1: General setup of closed loop feedback system with adaptation of controller parameters

As an example, consider the general closed loop adaptive feedback system as given in Fig. 1. Here, $r\left(t_{k}\right)$ is the (actual)

reference, $u\left(t_{k}\right)$ is the plant input and $y\left(t_{k}\right)$ is the plant output. The currently implemented controller parameters are denoted with $\check{\theta}\left(t_{k}\right)$ and $z^{-1}$ is the discrete time shift operator $\left(z^{-1} * t_{k}=t_{k-1}\right.$, with $*$ a discrete-time convolution). Assume that plant input $u\left(t_{k}\right)$ can be written as

$$
u\left(t_{k}\right)=K_{r}\left(\check{\theta}\left(t_{k}\right), z^{-1}\right) * r\left(t_{k}\right)+K_{y}\left(\check{\theta}\left(t_{k}\right), y\left(t_{k}\right), z^{-1}\right) .
$$

Then, for a given $u\left(t_{k}\right)$ and $y\left(t_{k}\right)$, the controller parameter dependent fictitious reference $r_{\text {fict }}\left(\theta, t_{k}\right)$ is given by

$$
r_{\text {fict }}\left(\theta, t_{k}\right)=K_{r}\left(\theta, z^{-1}\right)^{-1} *\left(u\left(t_{k}\right)-K_{y}\left(\theta, y\left(t_{k}\right), z^{-1}\right)\right) .
$$

As can be seen from (2) and (3), for $\theta=\check{\theta}\left(t_{k}\right), r_{\text {fict }}\left(\theta, t_{k}\right)$ exactly results in the actual reference $r\left(t_{k}\right)$, provided that $K_{r}(\theta)$ is Stably Causally-Left-Invertible. Of course, the restriction that $K_{r}\left(\theta, z^{-1}\right)$ is invertible limits the selection of candidate controllers. However, still a large class of controllers is available.

Let the controller structure be chosen such, that $r_{\text {fict }}\left(\theta, t_{k}\right)$ is affine in the controller parameters $\theta$. Furthermore, let $r_{\text {fict }}\left(\theta, t_{k}\right)$ depend on $u\left(t_{k}\right), y\left(t_{k}\right)$ and filtered versions thereof. Additionally, let $r_{\text {fict }}\left(\theta, t_{k}\right)$ depend on nonlinear functions of $y\left(t_{k}\right)$. Then, a general notation of $r_{\text {fict }}\left(\theta, t_{k}\right)$ is given by

$$
\begin{aligned}
r_{\text {fict }}\left(\theta, t_{k}\right) & =\left[\begin{array}{c}
u\left(t_{k}\right) \\
\Lambda_{u}\left(z^{-1}\right) * u\left(t_{k-1}\right) \\
\Lambda_{y}\left(z^{-1}\right) * y\left(t_{k}\right) \\
f\left(u\left(t_{k-1}\right), y\left(t_{k}\right), z^{-1}\right)
\end{array}\right]^{T}\left[\begin{array}{c}
\theta_{1} \\
\underline{\theta}_{2} \\
\underline{\theta}_{3} \\
\underline{\theta}_{4}
\end{array}\right] \\
& =w\left(u\left(t_{k}\right), y\left(t_{k}\right), z^{-1}\right)^{T} \theta,
\end{aligned}
$$

where $\Lambda_{u}\left(z^{-1}\right)$ and $\Lambda_{y}\left(z^{-1}\right)$ are vectors of stable linear filters and $f\left(u\left(t_{k-1}\right), y\left(t_{k}\right), z^{-1}\right)$ is a vector with nonlinear functions. For $\check{\theta}_{1} \neq 0$, (4) uniquely defines the control input $u\left(t_{k}\right)$.

The concept of a fictitious reference enables the evaluation of controllers, even if they were not in the loop at the time of the measurement, as is shown next.

\section{Unfalsification}

Given a desired performance specification, and exploiting the fictitious reference, a region can be constructed of controller parameters which are unfalsified by current measurement data.

Let the performance specification be defined as a timedependent maximum allowed tracking error $\Delta\left(t_{k}\right)$, as in [3]. Then the region of controller parameters, which is unfalsified by current measurement data at time $t_{k}$, is given by

$$
\begin{aligned}
\mathcal{U}\left(t_{k}\right)= & \left\{\theta \mid-\Delta\left(t_{k}\right) \leq\right. \\
\left.G_{m}\left(z^{-1}\right) * r_{\text {fict }}\left(\theta, t_{k}\right)-y\left(t_{k}\right) \leq \Delta\left(t_{k}\right)\right\} & \\
= & \{\theta \mid-1 \leq \\
& \left.\frac{G_{m}\left(z^{-1}\right) * w\left(u\left(t_{k}\right), y\left(t_{k}\right), z^{-1}\right)^{T}}{\Delta\left(t_{k}\right)} \theta-\frac{y\left(t_{k}\right)}{\Delta\left(t_{k}\right)} \leq 1\right\},
\end{aligned}
$$


with $G_{m}\left(z^{-1}\right)$ the desired closed loop dynamics. From (7) it is clear to see, that $\mathcal{U}\left(t_{k}\right)$ defines two parallel half-spaces in the controller parameter space $\theta$.

\section{E. Update Unfalsified set}

The region of controllers, that is unfalsified by all available measurement data (hence, including all past and present measurement data), is given by the intersection of the candidate controllers $\mathcal{E}\left(t_{k-1}\right)$ from section III-B (the controllers that are unfalsified by past measurement data) and the controllers $\mathcal{U}\left(t_{k}\right)$ from section III-D (the controllers that are unfalsified by the present measurement data).

To maintain an ellipsoidal Unfalsified set, the intersection $\mathcal{E}\left(t_{k-1}\right) \cap \mathcal{U}\left(t_{k}\right)$ is approximated by a minimum-volume outer-bounding ellipsoid $\mathcal{E}\left(t_{k}\right)$. Since $\mathcal{U}\left(t_{k}\right)$ defines two parallel half-spaces, this approximation can be computed analytically, as was shown in [7]. To compute $\mathcal{E}\left(t_{k}\right)$, define the variables

$$
\begin{aligned}
y_{k} & =\frac{y\left(t_{k}\right)}{\Delta\left(t_{k}\right)} \\
\phi_{k} & =\frac{G_{m}\left(z^{-1}\right) * w\left(u\left(t_{k}\right), y\left(t_{k}\right), z^{-1}\right)}{\Delta\left(t_{k}\right)} \\
g & =\phi_{k}^{T} \Sigma\left(t_{k-1}\right) \phi_{k} \\
a_{+} & =\max \left(\frac{y_{k}-\phi_{k}^{T} \theta_{c}\left(t_{k-1}\right)-1}{\sqrt{g}},-1\right) \\
a_{-} & =\max \left(\frac{-y_{k}+\phi_{k}^{T} \theta_{c}\left(t_{k-1}\right)-1}{\sqrt{g}},-1\right) .
\end{aligned}
$$

If $a_{+} a_{-} \geq 1 / p$ (Recall from (1) that $p$ is the number of controller parameters), $\mathcal{E}\left(t_{k-1}\right)$ is the minimum-volume outer-bounding ellipsoid of the intersection, hence, $\mathcal{E}\left(t_{k}\right)=$ $\mathcal{E}\left(t_{k-1}\right)$. Consequently, $\Sigma\left(t_{k}\right)=\Sigma\left(t_{k-1}\right)$ and $\theta_{c}\left(t_{k}\right)=$ $\theta_{c}\left(t_{k-1}\right)$, with $\Sigma\left(t_{k}\right)$ and $\theta_{c}\left(t_{k}\right)$ as in (1).

For $a_{+} a_{-}<1 / p$, if $a_{+} \neq a_{-}, \mathcal{E}\left(t_{k}\right)$ is defined by

$$
\begin{aligned}
\Sigma\left(t_{k}\right) & =\delta\left(\Sigma\left(t_{k-1}\right)-\frac{\sigma}{g} \Sigma\left(t_{k-1}\right) \phi_{k} \phi_{k}^{T} \Sigma\left(t_{k-1}\right)\right) \\
\theta_{c}\left(t_{k}\right) & =\theta_{c}\left(t_{k-1}\right)+\frac{\sigma\left(a_{+}-a_{-}\right)}{2 \sqrt{g}} \Sigma\left(t_{k-1}\right) \phi_{k},
\end{aligned}
$$

with

$$
\begin{aligned}
\delta= & \frac{p^{2}}{p^{2}-1}\left(1-\frac{a_{+}^{2}+a_{-}^{2}-\rho / p}{2}\right) \\
\sigma= & \frac{1}{p+1} . \\
& {\left[p+\frac{2}{\left(a_{+}-a_{-}\right)^{2}}\left(1-a_{+} a_{-}-\frac{\rho}{2}\right)\right] } \\
\rho= & \sqrt{4\left(1-a_{+}^{2}\right)\left(1-a_{-}^{2}\right)+p^{2}\left(a_{+}^{2}-a_{-}^{2}\right)^{2}} .
\end{aligned}
$$

If $a_{+}=a_{-}=a, \sigma$ in (16) becomes unbounded and, therefore, is defined by

$$
\lim _{a_{+} \rightarrow a_{-}} \sigma=\frac{1-p a^{2}}{1-a^{2}} .
$$

\section{F. Controller Selection}

A controller, that is unfalsified by the available measurement data, is to be selected to be inserted in the loop. Or in other words, one controller inside $\mathcal{E}\left(t_{k-1}\right) \cap \mathcal{U}\left(t_{k}\right)$ is to be implemented.

Consider the controller selection algorithm

$$
\check{\theta}\left(t_{k}\right)= \begin{cases}\check{\theta}\left(t_{k-1}\right) & \text { if }-1 \leq \gamma \leq 1 \\ \alpha \frac{-1-\gamma_{c}}{\gamma-\gamma_{c}} \check{\theta}\left(t_{k-1}\right)+ & \text { if } \gamma<-1 \\ \left(1-\alpha \frac{-1-\gamma_{c}}{\gamma-\gamma_{c}}\right) \theta_{c}\left(t_{k}\right) & \\ \alpha \frac{1-\gamma_{c}}{\gamma-\gamma_{c}} \check{\theta}\left(t_{k-1}\right)+ & \text { if } \gamma>1 \\ \left(1-\alpha \frac{1-\gamma_{c}}{\gamma-\gamma_{c}}\right) \theta_{c}\left(t_{k}\right) & \end{cases}
$$

with $\check{\theta}\left(t_{k-1}\right)$ the currently implemented controller parameters (as in (2)), and

$$
\begin{aligned}
\gamma & =\phi_{k}^{T} \check{\theta}\left(t_{k-1}\right)-y_{k} \\
\gamma_{c} & =\phi_{k}^{T} \theta_{c}\left(t_{k}\right)-y_{k} .
\end{aligned}
$$

For $|\gamma|>1, \check{\theta}\left(t_{k-1}\right) \notin \mathcal{U}\left(t_{k}\right)$ and, hence, $\check{\theta}\left(t_{k-1}\right)$ is falsified by current measurement data.

The parameter $\alpha \in[0,1]$ determines the stepsize of the switching algorithm. Choosing $\alpha=0$ corresponds to switching to the center of $\mathcal{E}\left(t_{k}\right)$, which is the point furthest from the bound of the Unfalsified set, but which might be considered as aggressive switching. To decrease aggressiveness, up to $\alpha=1$ might be chosen, which corresponds to a point on the boundary of $\mathcal{U}\left(t_{k}\right)$. In Fig. 2, the difference between $\alpha=0$ and $\alpha=1$ is schematically depicted.
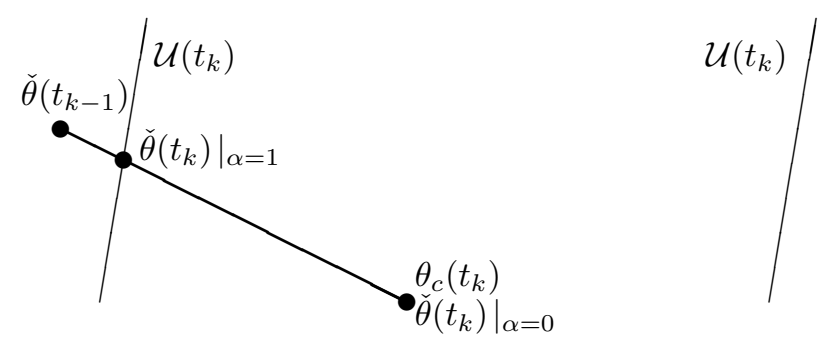

Fig. 2: Example of cautious $(\alpha=1)$ versus aggressive $(\alpha=$ 0) switching

\section{STABILITY OF EUC}

In the previous section, the Ellipsoidal Unfalsified Control algorithm was introduced. In this section, the stability of an adaptive control system with the EUC algorithm is addressed. It is shown that, with some extensions, the stability of the adaptive control system can be guaranteed. At the end of this section, the results are summarized in a theorem.

Ellipsoidal Unfalsified Control only considers the external, or input-output, behavior of a plant, in contrast to the internal, or state-space, behavior. This naturally leads to the stability concept of bounded-input bounded-output stability.

Definition 3 (BIBO stability): A system $G$ with input $v(t)$ and output $w(t)$ is bounded-input bounded-output (BIBO) 
stable, if, for every $v(t) \in\left\{|v(t)| \leq \alpha_{s}<\infty, \forall t\right\}$, it holds that $w(t) \in\left\{|w(t)| \leq \beta_{s}<\infty, \forall t\right\}$ for some constants $\alpha_{s}$ and $\beta_{s}$. Otherwise, the system is said to be unstable.

In [10] and [12], conditions are given for an adaptive control system to be stable. In short, these boil down to the next lemma

Lemma 1: Sufficient plant-model-free conditions to guarantee stability of an adaptive control system are:

1) The adaptive control problem is feasible

2) It is detected when a controller is destabilizing the adaptive control system, without assumptions on the plant. Controllers, that are demonstrably destabilizing from measurement data, are ineligible for implementation / discarded from the candidate set

3) The maximum number of controller switches is limited.

In the remainder of this section, the 3 conditions from Lemma 1 will be elaborated upon for Ellipsoidal Unfalsified Control. It should be noted that all considerations regard BIBO stability.

\section{A. Feasibility}

Condition 1 is fulfilled by assumption. The controller structure is chosen rich enough, such that it is likely that this assumption will be fulfilled. No information is available (to the authors knowledge) that predicts the feasibility of an adaptive control system, if no extensive plant-model is available.

\section{B. Discarding of demonstrably destabilizing controllers}

Condition 2 is enforced by considering only SCLI controllers, that, in combination with the performance specification, discard demonstrably destabilizing controllers.

1) SCLI: Consider the general notation of the EUC controller structure, as given in (4). Observe that, for $\left|u\left(t_{k}\right)\right|,\left|y\left(t_{k}\right)\right|,\left|f\left(u\left(t_{k-1}\right), y\left(t_{k}\right), z^{-1}\right)\right| \leq \alpha_{s}<\infty \forall t_{k}$, $\Lambda_{u}\left(z^{-1}\right), \Lambda_{y}\left(z^{-1}\right)$ stable, and $\left|\check{\theta}_{i}\right|<\infty \forall t_{k}, i=\{1,2,3,4\}$, $\check{\theta}_{1} \neq 0$, then $\left|r_{\text {fict }}\left(\theta, t_{k}\right)\right| \leq \beta_{s}<\infty \forall t_{k}$ for some $\alpha_{s}, \beta_{s}$. From this observation, it can be concluded that the fictitious reference signal is uniquely determined by past and present measurement data and that this mapping is stable. Therefore, the EUC controller structure (4) is Stably Causally-LeftInvertible.

2) $l_{\infty}$ performance specification: Consider the performance specification, as given in (6). For unfalsified controllers, it follows that

$$
\left|y(t)-G_{m}\left(z^{-1}\right) * r_{\text {fict }}\left(\theta, t_{k}\right)\right| \leq \Delta\left(t_{k}\right) .
$$

From Section IV-B. 1 it is concluded that $\left|r_{\text {fict }}\left(\theta, t_{k}\right)\right| \leq$ $\beta_{s} \forall t_{k}$ for some $\beta_{s}<\infty$. Then, for a stable reference model $G_{m}\left(z^{-1}\right)$, it holds that $\left|G_{m}\left(z^{-1}\right) * r_{\text {fict }}\left(\theta, t_{k}\right)\right|<\infty$. Furthermore, since $\Delta\left(t_{k}\right)<\infty \forall t_{k}$, for an unfalsified controller $K$ it holds that

$$
\begin{aligned}
\left|y\left(t_{k}\right)\right|-\mid G_{m}\left(z^{-1}\right) * & r_{\text {fict }}\left(\theta, t_{k}\right) \mid \\
& \leq\left|y(t)-G_{m}\left(z^{-1}\right) * r_{\text {fict }}\left(\theta, t_{k}\right)\right| \\
& \leq \Delta\left(t_{k}\right) \Rightarrow \\
\left|y\left(t_{k}\right)\right| \leq \mid G_{m}\left(z^{-1}\right) & * r_{\text {fict }}\left(\theta, t_{k}\right) \mid+\Delta\left(t_{k}\right)<\infty
\end{aligned}
$$

From (23) it can be concluded, that $\mid y(t)-G_{m}\left(z^{-1}\right) *$ $r_{\text {fict }}\left(\theta, t_{k}\right) \mid \leq \Delta\left(t_{k}\right)$ is a sufficient condition to not falsify the stability of controller $K$. Hence, for the controllers in the (True) Unfalsified set, it can be concluded that the BIBO stability is unfalsified by measurement data and that the EUC controller structure (4) and performance specification (6) discard controllers, that are demonstrably destabilizing from measurement data.

\section{Limited number of switches}

Ellipsoidal Unfalsified Control uses an ellipsoidal description of the Unfalsified set, which is continuous in the controller parameter space. Hence, an infinite number of candidate controllers is considered. The proof that condition 3 of lemma 1 is fulfilled for EUC is split in 2 parts: first it is shown that, with a minor constraint, a finite number of ellipsoidal Unfalsified sets can be guaranteed. Then it is shown that, again with a minor constraint, a finite number of controller switches per ellipsoid can be guaranteed. Hence, by combining these 2 parts, a finite number of overall controller switches can be guaranteed.

1) Limited number of ellipsoids: Note, that the volume of the ellipsoidal Unfalsified set is monotonically decreasing. Furthermore, the volume is lower bounded by $e$, the volume of the region $E$ by assumption of feasibility, see Section II. Here, it is shown that a maximum volume ratio between two consecutive ellipsoids implies a maximum number of ellipsoids.

\section{Decrease of volume}

To address the decrease in volume of two consecutive ellipsoids, consider the volume ratio

Lemma 2: [4, Lemma 3] The volume ratio $\delta_{V}\left(t_{k}\right)$ between two consecutive ellipsoids for $a_{+} \neq a_{-}$is given by

$$
\delta_{V}\left(t_{k}\right)=\sqrt{\delta^{p}(1-\sigma)} .
$$

Corollary 1: [4, Corollary 1] The volume ratio $\delta_{V}\left(t_{k}\right)$ between two consecutive ellipsoids is given by

$$
\delta_{V}\left(t_{k}\right)=\sqrt{\delta^{p}\left(1-\frac{1-p a^{2}}{1-a^{2}}\right)}
$$

for $a_{+}=a_{-}=a$.

From Lemma 2 it can be concluded that the volume of the ellipsoids decreases when $\delta^{p}(1-\sigma)<1$.

Conditions for a finite number of ellipsoids

The volume ratio between two consecutive ellipsoids is given in (24) and (25). Consider a maximum volume ratio $\delta_{V} \leq \nu$ for some positive $\nu<1$. From (24), it can be seen that a sufficient condition to ensure $\delta_{V} \leq \nu$ between two consecutive ellipsoids is to require that $a_{+} a_{-} \leq \epsilon(\nu) / p$ for 
some $\epsilon(\nu)<1$. The value of $\epsilon(\nu)$ can be derived from (24). It is observed, that for $\epsilon(\nu)$ close to 1 ,

$$
\arg \max _{a_{+} a_{-}=\epsilon(\nu) / p} \delta_{V}\left(t_{k}\right)=\left\{\begin{array}{l}
\left\{a_{+}=-1, a_{-}=-\epsilon(\nu) / p\right\} \\
\left\{a_{-}=-1, a_{+}=-\epsilon(\nu) / p\right\}
\end{array}\right.
$$

for a fixed $p$.

If $\epsilon(\nu) / p<a_{+} a_{-} \leq 1 / p$, the additionally falsified region is neglected and the Unfalsified set is not changed.

To express the maximum number of ellipsoidal Unfalsified sets, consider the volume $V\left(t_{0}\right)$ of the initial Unfalsified set, which is the largest possible volume of the Unfalsified set. The volume of the $n^{\text {th }}$ ellipsoid is upperbounded by $V\left(t_{0}\right) \nu^{n}$. Next, consider the smallest possible volume $e$ of the Unfalsified set, which is given by the volume of region $E$. Then the maximum number of ellipsoids $n_{\mathrm{e}}$ is limited by

$$
\begin{aligned}
\nu^{n_{\mathrm{e}}} & =\frac{e}{V\left(t_{0}\right)} \Rightarrow \\
n_{\mathrm{e}} & =\frac{\log \left(e / V\left(t_{0}\right)\right)}{\log (\nu)} .
\end{aligned}
$$

2) Limited number of switches per ellipsoid: To limit the number of controller switches, first observe that several controllers can be chosen consecutively within the same ellipsoid, as a function of the location of $\mathcal{U}\left(t_{k}\right)$. However, this can only continue while $a_{+} a_{-}>\epsilon(\nu) / p$, for otherwise the ellipsoid is to be updated. Next, observe that for controller selection algorithm (19), all consecutive controllers for a given ellipsoid are on the same line segment $\left[\ddot{\theta}\left(t_{k}-1\right), \theta_{c}\left(t_{k}\right)\right]$. By enforcing a minimum stepsize on the controller adjustments, this line segment can be divided in a maximum number of adjustments. Therefore, consider the additional constraint $\alpha \leq \epsilon_{\alpha}$ on parameter adjustment parameter $\alpha$ from (19), for some $\epsilon_{\alpha}<1$ :

$$
0 \leq \alpha \leq \epsilon_{\alpha}<1 .
$$

Then the maximum number of controller switches per ellipsoid $n_{\text {cspe }}$ is given by

$$
\begin{aligned}
\epsilon_{\alpha}^{n_{\text {cspe }}} & =\epsilon(\nu) / p \Rightarrow \\
n_{\text {cspe }} & =\frac{\log (\epsilon(\nu) / p)}{\log \left(\epsilon_{\alpha}\right)} .
\end{aligned}
$$

Remark: The maximum numbers of ellipsoidal Unfalsified sets and controller switches per ellipsoid, as derived above, are not concerned with an accurate prediction of the actual attainable number of controller switches. They merely serve as a demonstration of the upperbound on the number of controller switches.

\section{Summary and remarks}

From Lemma 1 and section IV-A through IV-C, the following theorem can be deduced:

Theorem 1 (Stability EUC): An Ellipsoidal Unfalsified Control System is BIBO stable, if

1) the adaptive control problem is feasible

2) the SCLI candidate controllers of (4) are considered, in combination with the $l_{\infty}$ performance specification (6)
3) the maximum number of controller switches is limited, by imposing an $\epsilon(\nu)<1$ on the update of the ellipsoid and an $\epsilon_{\alpha}<1$ on the controller update

Proof: In Section IV-B it is shown, that demonstrably destabilizing controllers are discarded, when considering the EUC controller structure (4) and the $l_{\infty}$ performance specification (6). From the feasibility of the adaptive control problem, it follows that there exists at all times a region $\supseteq E$ of candidate controllers, which is unfalsified. As the number of controller switches is limited by imposing $\epsilon(\nu)<1$ and $\epsilon_{\alpha}<1$, switching will eventually stop, resulting in a fixed controller that is unfalsified $\forall t$, hence, that is stable $\forall t$.

Since the preceding is true for any bounded $r(t)$, it can be concluded that the stability of the Ellipsoidal Unfalsified Control system is unfalsified for all bounded $r(t)$, and, hence, that the Ellipsoidal Unfalsified Control system is stable.

\section{SIMULATION}

In simulations, the effect of Ellipsoidal Unfalsified Control has been evaluated. Consider a fourth order plant, as is given by

$$
\left\{\begin{aligned}
\frac{d}{d t} x & =A x+B u \\
y & =C x
\end{aligned}\right.
$$

with

$$
\begin{aligned}
A & =\left[\begin{array}{cccc}
0 & 1 & 0 & 0 \\
-c / J_{1} & -d / J_{1} & c / J_{1} & d / J_{1} \\
0 & 0 & 0 & 1 \\
c / J_{2} & d / J_{2} & -c / J_{2} & -d / J_{2}
\end{array}\right] \\
B & =\left[\begin{array}{llll}
0 & 1 / J_{1} & 0 & 0
\end{array}\right]^{T} \\
C & =\left[\begin{array}{llll}
0 & 0 & 1 & 0
\end{array}\right] .
\end{aligned}
$$

The parameter-values are chosen as $J_{1}=1.56 \cdot 10^{-4}, J_{2}=$ $1.95 \cdot 10^{-4}, d=0.9 \cdot 10^{-3}$ and $c=8.64$. The plant is sampled at $1 \mathrm{kHz}$ with a zero order hold and an output disturbance with noise power $10^{-8}$ is present.

The controller structure $w\left(u\left(t_{k}\right), y\left(t_{k}\right), z^{-1}\right)$ is chosen as

$$
w\left(u\left(t_{k}\right), y\left(t_{k}\right), z^{-1}\right)=\left[\begin{array}{c}
u\left(t_{k}\right) \\
10^{-3} \frac{1+z^{-1}}{1-z^{-1}} u\left(t_{k}\right) \\
y\left(t_{k}\right) \\
10^{-3} \frac{1+z^{-1}}{1-z^{-1}} y\left(t_{k}\right) \\
y^{2}\left(t_{k}\right)
\end{array}\right] .
$$

The last element of $w\left(u\left(t_{k}\right), y\left(t_{k}\right), z^{-1}\right)$ is chosen, to underline that EUC is not limited to linear controllers. The maximum volume ratio is constrained by setting $\epsilon(\nu)=$ $0.99<1$. This corresponds to $\nu=0.999989$, which is close to, but still smaller than, 1 .

The reference to track is given by

$$
r\left(t_{k}\right)=\operatorname{sign}\left(\sin \left(0.5 \pi\left(t_{k}\right)\right)\right.
$$

and reference model

$$
G_{m}\left(z^{-1}\right)=\frac{2 \cdot 10^{-4}\left(z^{-1}+z^{-2}\right)}{1-1.96 z^{-1}+0.9604 z^{-2}} .
$$


The performance bound $\Delta\left(t_{k}\right)$ is given by

$$
\Delta\left(t_{k}\right)=0.02+e^{-t_{k}}
$$

Here, the lower bound on $\Delta\left(t_{k}\right)$ is included, to guarantee feasibility in the presence of a bounded output disturbance. The algorithm is initialized with

$$
\begin{aligned}
\Sigma(0) & =10^{4} \mathbb{I}_{5 \times 5} \\
\check{\theta}(0) & =\theta_{c}(0)=\left[\begin{array}{lllll}
100 & 0 & 1 & 0 & 0
\end{array}\right]^{T} .
\end{aligned}
$$

The initial value $\check{\theta}(0)$ corresponds to a P-controller with gain $0.01\left(\check{\theta}_{3} / \check{\theta}_{1}\right)$ (which, in fact, is destabilizing the system due to the phase lag caused by the zero order hold).

The stepsize of the controller parameter update is set to $\alpha=0$ (center of new ellipsoid, see (19)).

In Fig. 3, the tracking error $G_{m}\left(z^{-1}\right) * r\left(t_{k}\right)-y\left(t_{k}\right)$ of the EUC adaptive system is shown. Within 10 seconds the EUC algorithm has found a controller parameter set which is unfalsified for $\Delta=0.02$. In Fig. 4, controller parameters $\check{\theta}\left(t_{k}\right)$ are shown as a function of time, together with the center of the ellipsoidal Unfalsified set $\theta_{c}\left(t_{k}\right)$. If the tracking error of Fig. 3 is within the performance bounds, the controller parameters are unchanged. The center $\theta_{c}\left(t_{k}\right)$ on the other hand changes almost continuously.

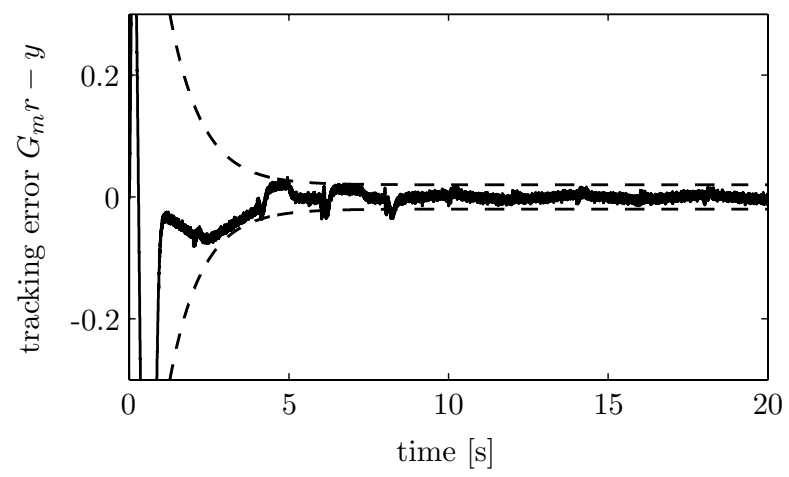

Fig. 3: Tracking error of plant (30) with EUC with control structure (34).
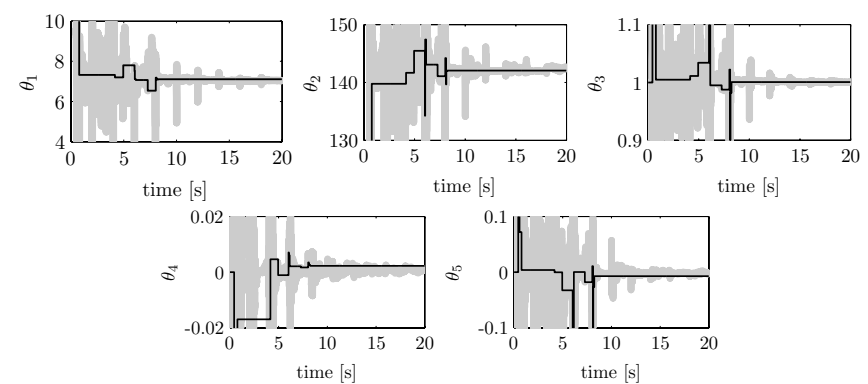

Fig. 4: Plot of the controller parameters $\check{\theta}\left(t_{k}\right)$ (black) as a function of time, together with the center of the ellipsoidal Unfalsified set $\theta_{c}\left(t_{k}\right)$ (grey).

\section{CONCLUSION}

Ellipsoidal Unfalsified Control is a data-driven, plantmodel-free control design method. A key issue in control design is the notion of stability. Although no a priori statements of the stability of a specific controller can be made for a data-driven control design method, it is shown that sufficient conditions exists to guarantee stability of the EUC adaptive control system.

In this paper, it is shown that sufficient conditions for Ellipsoidal Unfalsified Control to be stable are 1) feasibility of the adaptive control problem, 2) Stably Causally-LeftInvertible candidate controllers, that, in combination with the $\ell_{\infty}$ performance specification, discard demonstrable destabilizing controllers and 3) a limited maximum number of controller switches. The latter is accomplished, by imposing a minimum decrease on the volume of the Unfalsified set between two consecutive ellipsoids and a minimum stepsize on the controller adjustments.

In a simulation example with a fourth order system, the effectiveness of the proposed method is shown.

\section{REFERENCES}

[1] K.J. Åström and B. Wittenmark. Adaptive Control. Addison Wesley, Reading, Massachusetts, USA, second edition, 1995.

[2] F.B. Cabral and M.G. Safonov. Unfalsified model reference adaptive control using the ellipsoid algorithm. In Proc. Conf. Dec. \& Contr. pages 3250-3255, Maui, Hawaii, USA, December 2003.

[3] J.J.M. van Helvoort, B. de Jager, and M. Steinbuch. Unfalsified control using an ellipsoidal unfalsified region applied to a motion system. In Proc. IFAC World Congress, Prague, Czech Republic, July 2005.

[4] J.J.M. van Helvoort, B. de Jager, and M. Steinbuch. Ellipsoidal Unfalsified Control: Stability. In Proc. Amer. Contr. Conf., pages 40944099, Minneapolis, Minnesota, USA, June 2006.

[5] R.L. Kosut. Unfalsified nonlinear adaptive control. In Proc. Conf. Dec. \& Contr., pages 1243-1247, Sydney, Australia, December 2000.

[6] A. Paul. Multi-Controller Adaptive Control (MCAC): Cost Detectability, Stability and some Applications. $\mathrm{PhD}$ thesis, University of Southern California, Los Angeles, August 2005.

[7] L. Ros, A. Sabater, and F. Thomas. An ellipsoidal calculus based on propagation and fusion. IEEE Trans. Systems Man Cybernet., 32(4):430-442, August 2002.

[8] M.G. Safonov and F.B. Cabral. Fitting controllers to data. Systems Control Lett., 43(4):299-308, July 2001.

[9] M.G. Safonov and T.-C. Tsao. The unfalsified control concept and learning. IEEE Trans. Automatic Control, 42(6):843-847, June 1997.

[10] M. Stefanovic, A. Paul, and M.G. Safonov. Safe adaptive switching through infinite controller set: Stability and convergence. In Proc. IFAC World Congress, Prague, Czech Republic, July 2005.

[11] T.-C. Tsao and M.G. Safonov. Unfalsified direct adaptive control of a two-link robot arm. In Proc. Conf. Contr. Appl., pages 680-686, Kohala Coast-Island of Hawaii, Hawaii, USA, August 1999.

[12] R. Wang, A. Paul, M. Stefanovic, and M.G. Safonov. Cost-detectability and stability of adaptive control systems. In Proc. Conf. Dec. \& Contr. and Eur. Contr. Conf., pages 3584-3589, Seville, Spain, December 2005. 